\title{
Near-infrared fluorescent nanoprobes for highly sensitive cyanide quantification in natural waters
}

Pablo Llano-Suáreza, Diego Bouzas-Ramosa, José M. Costa-Fernández ${ }^{a}$, Ana Soldadob, María Teresa Fernández-Argüelles ${ }^{\text {a,* }}$

${ }^{a}$ Department of Physical and Analytical Chemistry, University of Oviedo, Oviedo (Asturias), Spain

${ }^{b}$ Department of Animal Nutrition, Grassland and Forages, Regional Institute for Research and AgroFood Development (SERIDA), Villaviciosa (Asturias), Spain

Corresponding author: E-mail: fernandezteresa@uniovi.es Telephone: +34985103513

Pablo Llano-Suárez: 0000-0002-8795-7870

Diego Bouzas-Ramos: 0000-0002-8063-9589Jose M. Costa-Fernández: 0000-0002-8671-5300

Ana Soldado: 0000-0003-2483-9620

María Teresa Fernández-Argüelles: 0000-0002-5191-3024 


\begin{abstract}
Near infrared (NIR) emitting $\mathrm{Ag}_{2} \mathrm{~S}$ quantum dots have been synthesized, characterized and evaluated for chemical sensing applications. After their optical characterization, it was observed that the $\operatorname{Ag}_{2} \mathrm{~S}$ quantum dots present both, excitation and emission in the NIR region, and an excellent quantum yield of $33.2 \%$. These features are of great value for many biological applications, since autofluorescence of biological tissues or cells is minimized, and also for environmental applications, where other fluorescent concomitant species with excitation and emission in the ultraviolet-visible region might be present.

Different purification procedures were evaluated in order to obtain a stable and homogeneous population of nanoparticles, which is necessary to perform quantitative analysis (e.g.: mass spectrometry-based applications), as well as to obtain a narrow NIR emission spectrum for optical applications.

Comprehensive characterization using X-ray diffraction, transmission electron microscopy, and asymmetric flow field flow fractionation coupled to inductively coupled plasma-mass spectrometry has been performed to obtain parameters not easily achieved and of great interest in different research areas, such as the nanoparticle concentration NIR-emitting nanoparticles, and the surface ligand density, which directly affects to the interactions of the nanoparticles with their close environment, including unspecific adsorptions, cellular uptake, macrophage interaction, etc.

Finally, the capability for sensing analytes of environmental interest based on direct-interactions of a reactive compound with the surface of the nanoparticle has been also evaluated. Quenching of the NIR emission upon interaction of the $\operatorname{Ag}_{2} \mathrm{~S}$ quantum dots with cyanide ions was observed. Hence, a rapid, selective and highly sensitive methodology was developed for the detection of cyanide in natural waters.
\end{abstract}

\title{
Keywords
}

Silver Sulfide, Near Infrared, Quantum Dot, Fluorescence, Sensing, Cyanide 


\section{Introduction}

Quantum Dots (QDs) are semiconductor nanocrystals that present excellent photoluminescent properties. Usually ranging between 1-10 nanometers, QDs are subject to quantum confinement effects due to their size being smaller than their exciton Bohr radius [1,2]. As a result, QDs have exceptional features that include high quantum yield fluorescence with narrow emission and wide absorption spectra, with very low photobleaching effect and high photostability. Nevertheless, the most studied QDs are those containing heavy metals, such as $\mathrm{Cd}$, and with excitation and emission wavelengths in the UV and Vis region respectively.

The development and study of novel QDs emitting in the NIR region is a very active research topic, because fluorescent background signals can be reduced, giving rise to an increase in the sensitivity. This is of enormous potential interest due to many biological species emit on the ultraviolet-visible range (e.g.: cell autofluorescence), interfering with the signal of conventional luminescent tags, while these interferences are absent in the infrared range. Moreover, when using NIR light, deeper penetration of the excitation source can be achieved in biological tissues, which is an enormous advantage in biomedical applications. Additionally, in other analytical fields such as the environmental area, the presence of fluorescent compounds in a certain sample, typically emitting in the UV-Vis range, can also produce an interferent effect that is avoided when the detection is performed in the NIR region. Besides these advantages related to their optical features, another trend is to develop QDs that do not contain toxic heavy metals, including $\mathrm{Cd}^{2+}, \mathrm{Hg}^{2+}, \mathrm{Pb}^{2+}$, that hinder the real application of luminescent QDs in biomedical applications [3]. In this sense, the synthesis and evaluation of the optical features of NIR II QDs, based on I-IV semiconductors $\left(\mathrm{Ag}_{2} \mathrm{~S}, \mathrm{Ag}_{2} \mathrm{Se}\right.$, and $\left.\mathrm{Ag}_{2} \mathrm{Te}\right)$ is being explored as a promising alternative to traditional NIR I type QDs, since they present excellent optoelectronic properties, including high luminescent quantum yields, good photostability, and low cytotoxicity [4].

Nowadays, in order to develop successful bioanalytical applications it is demanded to have reliable information about their elemental composition, size, shape, surface chemistry, and nanoparticle concentration [5]. Hence, to characterize the nanoparticles (NPs), a wide variety of complementary techniques are typically employed, including spectrophotometry, spectrofluorimetry, dynamic light scattering, transmission electron microscope, etc. However, there are certain applications that require a deeper characterization of the nanoparticles. For instance, it is very important to estimate the concentration of nanocrystals in solution, in terms of the number of nanoparticles per unit volume, in order to assess reliable quantitative analytical or bioanalytical applications based on NPs. In this sense, it is also very important to perform an appropriate purification of the synthesized nanoparticles to remove any excess of the precursors employed for the synthesis or the presence of agglomerates that would affect negatively to quantitative analytical applications. Moreover, the surface ligand density is also a key parameter that should be known because surface ligands are those that first interact with their close environment, and are also responsible of the colloidal stability of the NPs, affecting to aspects such as cellular uptake of the NPs, macrophage-nanoparticle interactions, unspecific adsorptions, stability in biological media, etc. [6]. 
In this work, highly luminescent $\mathrm{Ag}_{2} \mathrm{~S}$ nanoparticles with excitation and emission in the infrared region will be synthesized and deeply characterized by developing a methodology based on the use of complementary techniques that allow to estimate the nanoparticle number concentration and the surface ligand density, both key parameters for future viability of their applications at real samples analysis. Because the luminescence of these nanoparticles is directly related to their active surface, any changes in the surface produces a change in the luminescence. Therefore, the potential applicability of these nanoparticles for chemical sensing was evaluated to detect cyanide in natural waters. The proposed sensing method is based on the quenching effect produced in the fluorescence emission of silver sulfide quantum dots.

Cyanide is a widespread toxic ion, present in natural sources like many seeds, such as those of apricot or apple, and coming from some anthropogenic sources, like tobacco smoke, building fires or the gold mining industry, where cyanide is used as a complexing agent to dissolve and separate gold from ore. According to the World Health Organization (WHO), "The toxic effects of cyanide ion in humans and animals are generally similar and are believed to result from inactivation of cytochrome oxidase and inhibition of cellular respiration and consequent histotoxic anoxia" [7]. The heart, lungs and central nervous system are therefore particularly affected. Due to its toxicity, and because of the inherent risk of leaks or spillage associated with its extensive industrial use, it is extremely important to have fast, reliable methodologies that can quantify cyanide below the levels set by public safety organizations. The maximum tolerable levels in drinking water are set at $1.9 \times 10^{-5} \mathrm{M}$ by the WHO [8], $7.7 \times 10^{-6} \mathrm{M}$ by the US EPA [9] and Health Canada [10], and $1.9 \times 10^{-6} \mathrm{M}$ by the European Union [11]. More restrictive still are the Australian and New Zealand Environmental and Conservation Council (ANZECC) levels for freshwater and marine water, set at $1.5 \times 10^{-7} \mathrm{M}$ and $7.7 \times 10^{-8} \mathrm{M}$, respectively [12]. Such strict guidelines require either complex, time-consuming methodologies with preconcentration steps, or extremely sensitive approaches.

\section{Material and methods}

\section{Materials and Reagents}

All chemical reagents were of analytical grade and used as received without further purification. 3Mercaptopropionic Acid (3-MPA, 99+\%), Silver Nitrate (>99\%), Sodium Sulfide Nonahydrate ( $\geq 98 \%)$, Sodium Hydroxide, Platinum Octaethyl-Porphirin (PtOEP, 98\%), TRIZMA Base, Sodium Hydroxide and the salts of the different ions studied were purchased from Sigma-Aldrich (https://www.sigmaaldrich.com), part of Merck KGaA, Darmstadt, Germany. TRIZMA HCl was purchased from Acros Organics (www.acros.com), and Acetic Acid Glacial was purchased from Fisher (www.thermofisher.com), both part of Thermo Fischer Scientific, Geel, Belgium.

\section{Synthesis of Silver Sulfide Quantum Dots}

Silver Sulfide Quantum Dots were synthesized following a similar approach to that developed by Hocaoglu et al [13] with some modifications. Briefly, $0.14 \mathrm{~g}$ of 3-mercaptopropionic (3-MPA) acid were 
dissolved in $50 \mathrm{~mL}$ of deionized water and the $\mathrm{pH}$ was adjusted to 7.5 using sodium hydroxide and acetic acid $1 \mathrm{M}$ solutions. After that, $42.5 \mathrm{mg}$ of silver nitrate was added, and the $\mathrm{pH}$ was readjusted to 7.5. This solution was poured into a three-necked flask covered with aluminum film in order to minimize the exposure of the contents to the sunlight, and deoxygenated with argon. The flask was then heated up to $50^{\circ} \mathrm{C} .13 .9 \mathrm{mg}$ of sodium sulfide were then dissolved in $20 \mathrm{~mL}$ of deionized water, and deoxygenated using argon. This solution was slowly added into the three-necked flask under vigorous mechanical stirring, where it was allowed to react for 7 hours at $50^{\circ} \mathrm{C}$. The obtained solution was purified using 3$\mathrm{KDa}$ Amicon-Ultra centrifugal filters from Merck KGaA, Darmstadt, Germany (www.merckmillipore.com/), washing with ultrapure milli-Q water several times. To further purify the solution, 0.45 and $0.22 \mu \mathrm{m}$ PVDF syringe filters were also employed. The QD solution was then stored in the dark at $4^{\circ} \mathrm{C}$. Good reproducibility between batches after the synthesis of Ag2S QDs in terms of size, concentration and optical properties of the NPs was observed.

\section{Purification of Silver Sulfide QDs}

One of the most critical parameters in the development of applications based on the use of nanoparticles is the purity of the obtained colloidal dispersions. In order to obtain a high purity of nanoparticles, minimizing the presence of excess of the precursors employed for the synthesis or possible nanoparticle aggregates, different methodologies were tested: simple filtration using 0.45 and $0.22 \mu \mathrm{m}$ PVDF syringe filters, centrifugation (testing both the supernatant and the redissolved precipitate), the use of AmiconUltra centrifugal filters (testing both the retained and the filtered fractions), and the combination of centrifugation / Amicon filtering with syringe filtering.

\section{Instrumentation}

UV-Vis absorption measurements were performed with a GENESYS 10S UV-Vis Spectrophotometer (Thermo Scientific, Spain, http://www.thermofisher.com/). Photoluminescence properties of the synthesized QDs were studied using a Varian Cary Eclipse Fluorescence Spectrometer (Varian Iberica, Madrid, Spain, https://www.agilent.com/) equipped with a xenon discharge lamp (peak power equivalent to $75 \mathrm{~kW}$ ), a Czerny-Turner monochromator and photomultiplier tube detector (Model R-298). Fluorescence spectra were recorded using a fixed excitation wavelength of $750 \mathrm{~nm}$ with both, excitation and emission slits widths of $10 \mathrm{~nm}$. All measurements were made at constant temperature $\left(20{ }^{\circ} \mathrm{C}\right)$ and atmospheric pressure, using quartz cuvettes (Hellma, Germany, www.hellma-analytics.com/).

The presence of the expected reaction product was confirmed via Fourier transform infrared (FTIR) spectroscopy, using a Varian 670 IR FT-IR spectrometer (Varian Iberica, Spain, https://www.agilent.com/). Data have been acquired within the spectral range of 4000 to $600 \mathrm{~cm}^{-1}$ using attenuate total reflectance measurements.

X-Ray Diffraction (XRD) analysis was performed using an X'Pert Pro MPD instrument from PANalytical (www.panalytical.com/), with a goniometer radius $240 \mathrm{~mm}$ equipped with a PIXcel detector, $14^{\circ}$ divergence slit, $0.04 \mathrm{rad}$ primary and secondary Soller slits and a Ni $\mathrm{K}_{\alpha}$ filter. Samples were prepared by lyophilization, followed by a 24 -hour drying step at $180^{\circ} \mathrm{C}$. Then, they were loaded by depositing the QDs powder on a silica zero background sample holder (ZBH). XRD patters from 5 to $70^{\circ} 2 \theta$ were 
recorded at room temperature using $\mathrm{Cu} \mathrm{K}$ radiation $(\lambda=1.5418 \dot{\mathrm{A}})$ with the following measurement conditions: tube voltage of $45 \mathrm{kV}$, tube current $40 \mathrm{~mA}$, scan mode with a step size of $0.0131^{\circ} 2 \theta$ and a counting time of $180 \mathrm{~s}$.

Transmission electron microscopy (TEM) experiments were carried out on a JEOL-2000EX II (JEOL, Tokyo, Japan, https://www.jeol.co.jp/) to study the size of the QDs. Additionally, High Resolution Transmission Electron Microscopy (HRTEM) images were also obtained using a JEOL JEM 2100 (JEOL, Tokyo, Japan) instrument, to study both size and composition via Energy-dispersive X-ray spectroscopy (EDX). HRTEM operated at an accelerating voltage of $200 \mathrm{kV}$ and was equipped with an ultrahigh-resolution pole piece that provided a point-resolution better than $0.19 \mathrm{~nm}$. Samples were prepared by placing several drops of diluted QDs solution in ultrapure water onto a carbon-coated copper TEM grid and then allowed to air-dry before loading in the microscope.

In order to study the purity of the products obtained from the synthesis of the QDs, Asymmetric FlowField Flow Fractionation (AF4) was coupled to inductively coupled plasma mass spectrometry (ICP-MS). Separation was carried out on a Postnova AF2000 MultiFlow FFF (Postnova Analytics Inc., Landsberg, Germany, www.postnova.com/), using the separation experimental conditions summarized in Table 1. After separation, silver elemental analysis was performed using inductively coupled plasma mass spectrometry (ICP-MS). For this purpose, a Triple Quad (Agilent 8800 ICP-QQQ, Tokyo, Japan, https://www.agilent.com/) using a concentric nebulizer with double-pass glass spray chamber Scott type. Operation conditions for the ICP-MS analysis were optimized using a tuning solution. Elemental Ag was measured in on-mass MS/MS mode $\left(107 \mathrm{Ag}^{+}\right)$. The integration time for each of the targeted isotopes was $100 \mathrm{~ms}$.

Table 1 Asymmetric Flow Field Flow Fractionation operation conditions

\begin{tabular}{|l|l|}
\hline Parameter & Conditions \\
\hline Mobile Phase & Ammonium Acetate $0.15 \mathrm{gL}-1, \mathrm{pH} 10.5$, TWEEN 20 0,06 g/l \\
\hline Injection time (min) & 3 \\
\hline Transition time (min) & 1 \\
\hline Tip flow $(\mathrm{ml} / \mathrm{min})$ & 0.2 \\
\hline Crossflow $(\mathrm{ml} / \mathrm{min})$ & 2.5 \\
\hline Detector flow $(\mathrm{ml} / \mathrm{min})$ & 0.6 \\
\hline Membrane type & Regenerated cellulose with $10 \mathrm{kDa}$ cut-off \\
\hline Channel Dimmensions & $29 \mathrm{~cm}$ length and 500 $\mu \mathrm{m}$ thickness \\
\hline Injection volume $(\mu \mathrm{l})$ & 20 \\
\hline
\end{tabular}

\section{Results and Discussion}

Silver Sulfide Quantum Dots characterization 
TEM images showed that the obtained $\mathrm{Ag}_{2} \mathrm{~S}$ QDs are spherical nanoparticles. After plotting a TEM histogram to estimate the average diameter of the QDs, the size of the nanoparticles turned out to be $4 \pm 1$ $\mathrm{nm}(\mathrm{n}=120)$. HRTEM was also carried out on the synthesized particles (see Figure 1), showing wellresolved equidistant lattice fringes. The distance between the fringes was measured in different images showing different nanoparticle orientation, and were estimated to be $4 \AA, 7 \AA$ and $8 \AA$, which are in agreement with the lattice parameters of $\alpha$-monoclinic $\mathrm{Ag}_{2} \mathrm{~S}$, which are $\mathrm{a}=4.23 \AA \mathrm{A}=6.91 \AA$, and $\mathrm{c}=8.29$ $\AA$.

Moreover, in order to further assess the chemical composition of the synthesized $\mathrm{Ag}_{2} \mathrm{~S}$ QDs, EDX analysis was carried out, and results obtained confirm the presence of $\mathrm{Ag}$ and $\mathrm{S}$, together with the presence of $\mathrm{C}$ and $\mathrm{O}$ due to the ligands, and with the presence of $\mathrm{Na}$ and $\mathrm{Cu}$ attributed to impurities derived from the precursors of the nanoparticles and to the copper grid employed to hold the sample in the HRTEM microscope.

$<$ Figure 1 near here>

Once the $\mathrm{Ag}_{2} \mathrm{~S}$ QDs were synthesized and purified, FT-IR analysis was carried out to assess the binding of 3-MPA ligands to the silver sulfide core (see Figure 2). The FT-IR spectra shows a broad feature in the range $3300-2500 \mathrm{~cm}^{-1}$ that overlaps the $\mathrm{C}-\mathrm{H}$ stretching region and with a secondary absorption close to $2600 \mathrm{~cm}^{-1}$, characteristic for hydrogen-bonded $\mathrm{O}-\mathrm{H}$ of the carboxylic acid. Other bands that are associated with the $\mathrm{C}-\mathrm{O}$ and $\mathrm{O}-\mathrm{H}$ and tend to be less pronounced are located in the ranges $1320-1210 \mathrm{~cm}^{-1}$ (C-O stretch) and 960-850 $\mathrm{cm}^{-1}$ (hydrogen bonded $\mathrm{O}-\mathrm{H}$ out of plane bending). The $\mathrm{C}=\mathrm{O}$ absorption is almost always one of the most characteristic in the entire spectrum, with bands located between $1610-1550 \mathrm{~cm}^{-1}$ and $1420-1300 \mathrm{~cm}^{-1}$. The asymmetric/symmetric stretch vibration for methylene are located at 2935 2915/2865-2845 respectively. The thio group (C-S) tends to give rise to very weak absorptions, the band assigned is located at $710-685 \mathrm{~cm}^{-1}$. Moreover, the absence of the characteristic band of the S-H band present in thiols, between $2600-2550 \mathrm{~cm}^{-1}$, confirms that there is no unbound free 3-MPA ligand after purification of the nanoparticles [14].

$<$ Figure 2 near here>

Powder XRD analysis was also employed to study the structure of the synthesized $\mathrm{Ag}_{2} \mathrm{~S}$ QDs. For this purpose, the QDs were first lyophilized and then a 24-hour drying step was carried out at $180^{\circ} \mathrm{C}$. Results obtained can be observed in Figure 3. Identification of the phases present in the sample was carried out by searching different crystalline databases (Fiz Karlsruhe ICSD Ed. Nov-2016, CSD), and assigning the different Bragg peaks to the corresponding likely compound. The presence of the expected $\alpha$-monoclinic $\mathrm{Ag}_{2} \mathrm{~S}$ (ICSD 98-004-4507) was confirmed. Additional phases present in the sample correspond to reagent impurities $\left(\mathrm{AgNO}_{3}\right.$ and $\mathrm{Na}_{2} \mathrm{~S}$, indexed as 98-000-0374 and 98-065-6376). The remaining Bragg peaks could be explained as product impurities, likely as a result of the thermal treatment of the sample to remove the humidity in order to perform the measurement in the XRD instrument, corresponding to the crystalline phases $\mathrm{Ag}_{2} \mathrm{C}_{9} \mathrm{H}_{4} \mathrm{~N}_{2} \mathrm{O}_{3}$ and $\mathrm{Ag}_{2} \mathrm{C}_{7} \mathrm{H}_{11} \mathrm{~N}_{3} \mathrm{O}_{5}$ (Indexed in the open COD database as 96-151-7613 and 96-451-1864, respectively).

$<$ Figure 3 near here> 


\section{Absorption and photoluminescence characterization of silver sulfide QDs}

The synthesized $\mathrm{Ag}_{2} \mathrm{~S}$ QDs were dispersed in Milli-Q water, and optical measurements were performed in the optimum instrumental conditions. UV-Vis spectrum of $\mathrm{Ag}_{2} \mathrm{~S}$ QDs shows that absorbance is increased at shorter wavelengths (Figure 4). Nevertheless, studies performed in this research work demonstrated that 3-MPA produces a broad band at such wavelengths. In fact, a deeper study showed that there is a small shoulder with an absorption peak centered around $750 \mathrm{~nm}$ (see Figure 4 blue line). Moreover, when recording the luminescence excitation spectrum, a noticeable peak with a maximum at $750 \mathrm{~nm}(\lambda \mathrm{ex})$ is also observed, this wavelength was selected as optimum excitation wavelength. A very intense, narrow and symmetric emission spectrum with a maximum emission wavelength centered at $800 \mathrm{~nm}(\lambda \mathrm{em})$ was obtained. These excitation and emission properties, both in the NIR region, are an advantage when using these QDs, because they can be employed for a wide range of applications where the UV-Vis fluorescence (e.g.: cell autofluorescence, presence of other fluorophores in environmental samples, etc) is a limitation. Despite reports suggesting that some Ag2S QDs could also present luminescence in the NIR-II region (1000-1320 nm), we did not register significant photoluminescence emission for the heresynthesised NPs in that spectral region. Therefore, for the development of the here-proposed methodology for cyanide quantification we have selected the NIR-I region, that can be easily measured using common UV-VIS photoluminescence instruments.

To study the temporal stability of the obtained QDs in aqueous solution, the fluorescence emission has been measured for 6 months. For this purpose, and to minimize any instrumental variance, the intensity was compared to the emission obtained for Rhodamine standard solution, a widely used fluorophore. Results obtained have shown that the optical properties of the $\operatorname{Ag}_{2} \mathrm{~S}$ QDs are stable in aqueous solution up to six months without any loss of their optical properties.

$<$ Figure 4 near here>

The fluorescent quantum yield value (QY) of $\mathrm{Ag}_{2} \mathrm{~S}$ QDs was calculated by using the comparative William's method [15].For this purpose, a fluorophore with known quantum yield, platinum octaethylporphyrin (PtOEP) was selected as a reference and the QY value was calculated according to equation 1.

$$
Q Y_{s}=\frac{F_{s} I_{r} Q Y_{r}}{F_{r} I_{s}} \frac{n_{s}{ }^{2}}{n_{r}{ }^{2}}
$$

Equation1

where, "Fs" is the integrated fluorescence emission of the sample, "Fr" is the integrated fluorescence emission of the reference (platinum octaethylporphyrin), "Is" is the absorbance at the excitation wavelength of the sample, "Ir" is the absorbance at the excitation wavelength of the reference, "QYs" is the quantum yield of the sample, and "QYr" is the quantum yield of the reference fluorophore (platinum octaethylporphyrin QY $=41.5 \%$ ). Since the solvent is different for the reference fluorophore (PtOEP is prepared in toluene) and the sample (QDs are dissolved in water), the equation includes the refractive 
indexes of sample " $\mathrm{n}_{\mathrm{s}}$ " and reference " $\mathrm{n}_{\mathrm{r}}$ ". Results obtained gave rise to a very good fluorescent QY of $33.2 \%$, demonstrating that these $\mathrm{Ag}_{2} \mathrm{~S}$ QDs emit a very intense NIR luminescence.

\section{Nanoparticle purification}

As explained before, the purity of the synthesized QDs is critical for the development of analytical applications. For this purpose, various purification processes were tested (see Materials and Methods section). Purification results obtained were evaluated in terms of the Full Width at Half Maximum (FWHM) of the emission spectrum, the intensity of the emission, and the fractogram obtained using AF4 coupled to ICP-MS. The nanoparticles that showed a highest emission intensity and provided the narrowest FWHM were those purified by using 3-kDa Amicon-Ultra centrifugal filters, washed with ultrapure milli-Q water several times, and followed by a second filtration using 0.45 and $0.22 \mu \mathrm{m}$ PVDF syringe filters consecutively. Then, the QD solution was stored in the dark at $4^{\circ} \mathrm{C}$.

In order to ensure the efficiency of the purification procedure the hyphenation of Asymmetric Flow-Field Flow Fractionation (AF4) to on-line ICP-MS analysis was performed. This combination allows performing the elemental silver quantification after separation of the nanoparticles from any other possible excess of unreacted precursors, by-products or nanoparticles aggregates derived from the synthesis of the QDs. The experimental conditions employed in this assay where soft enough avoid destroying the nanoparticles.

As can be seen in the fractogram (Figure 5), the "void peak" attributed to the injection of the sample appears at $300 \mathrm{~s}$, and afterwards there is a narrow and intense peak with a 1:1 Ag:S molar ratio $(\mathrm{t}=6.5$ min). This peak corresponds to a single population of $\mathrm{Ag}_{2} \mathrm{~S}$ nanoparticles, but the expected results were associated to the molar ratio of $\mathrm{Ag}_{2} \mathrm{~S}$ (2:1). To explain these findings, it is necessary to remark that there is an excess of sulphur coming from the 3-MPA shell that contribute to these 1:1 molar ratio. A second population of the unpurified QDs (Figure 5, grey line) can be observed in the fractogram, it corresponds to impurities. These are eliminated by using the purification procedure with a 3-kDa Amicon ultracentrifugal filter because they are not observed in the fractogram of the purified QDs (Figure 5, black line). Finally, a third population, with a 1:2 Ag:S molar ratio, is attributed to $\mathrm{Ag}_{2} \mathrm{~S}$ NPs with higher ligand density. This estimation will be described and discussed in a following section. The AF4 recoveries for the unpurified and purified NPs are nearly quantitative $(95 \pm 1 \%)$ for both purified and raw NPs.

$<$ Figure 5 near here>

\section{Estimation of silver sulfide QDs concentration.}

It is well known that a very important parameter for the development of bioanalytical applications based on the use of nanoparticles is the particle concentration. Hence, using the size estimated by HRTEM, the stoichiometry previously obtained from the XRD analysis, and the atomic mass of silver and sulfur, it is 
possible to calculate the nanoparticle number concentration in solution as QD molarity after ICP-MS analysis using the following equation:

$$
C_{N P}=\frac{N_{N P}}{N_{A} \times V}
$$

Where $\mathrm{C}_{\mathrm{NP}}$ is the molar concentration of nanoparticles in solution, $\mathrm{N}_{\mathrm{NP}}$ is the number of nanoparticles, $\mathrm{N}_{\mathrm{A}}$ is the number of Avogadro and $\mathrm{V}$ the volume of the solution. The number of nanoparticles $\left(\mathrm{N}_{\mathrm{NP}}\right)$ can be obtained using both, the total nanoparticle volume $\mathrm{V}_{\mathrm{t}, \mathrm{NP}}$ and the volume of each nanoparticle $\mathrm{V}_{\mathrm{NP}}$ (equation 3):

$$
N_{N P}=\frac{V_{t, N P}}{V_{N P}}
$$

Equation 3

The volume of each nanoparticle can be easily estimated assuming that QDs are spherical shaped (it was previously demonstrated with the TEM images), and the total volume can be calculated using the density and mass of $\mathrm{Ag}_{2} \mathrm{~S}$ in solution following equations 4 and 5 respectively:

$$
V_{N P}=\frac{4}{3} \pi\left(\frac{d_{c}}{2}\right)^{3} \quad V_{t, N P}=\frac{m_{N P}}{\rho_{A g_{2} S}} \quad \text { Equation } 4 \text { and } 5
$$

where $d_{c}$ is the diameter of the nanoparticles, $\rho_{\mathrm{Ag} 2 \mathrm{~S}}$ is the density of silver sulfide $\left(\rho_{\mathrm{Ag} 2 \mathrm{~S}}=7,23 \mathrm{~g} / \mathrm{cm}^{3}\right)$, and $\mathrm{m}_{\mathrm{NP}}$ is the sum of $\mathrm{m}_{\mathrm{Ag}}$ and $\mathrm{m}_{\mathrm{S}}$, which are the mass of silver and sulfur in solution respectively. $\mathrm{m}_{\mathrm{Ag}}$ is obtained directly from the elemental ICP-MS measurements while, since sulfur is difficult to quantify by ICP-MS, $\mathrm{m}_{\mathrm{S}}$ is indirectly calculated by the following equation 6 :

$$
m_{S}=\frac{m_{A g} \times M_{S}}{2 \times M_{A g}}
$$

Equation 6

Hereby, $\mathrm{M}_{\mathrm{S}}$ is the molar mass of sulfur and $\mathrm{M}_{\mathrm{Ag}}$ the molar mass of silver, the number two is the molar ratio of silver to sulfur in the $\mathrm{Ag}_{2} \mathrm{~S}$ nanoparticles, as confirmed by XRD. The $\mathrm{m}_{\mathrm{Ag}}$ was estimated by using the AF4-ICP-MS. This hyphenated technique allowed to quantify the Ag content confirming that all Ag quantified came from $\mathrm{Ag}_{2} \mathrm{~S}$ nanoparticles (and not from unreacted silver precursor). After that, those previously described equations were employed satisfactorily, and the nanoparticle number concentration obtained was of $2 \cdot 10^{-7} \mathrm{M}$ of $\mathrm{Ag}_{2} \mathrm{~S}$ QDs.

\section{Estimation of surface ligand density}

After calculating silver and nanoparticle concentration, the following step was to estimate the number of silver atoms per nanoparticle using the equation 7

$$
\frac{[\mathrm{Ag}]}{[\mathrm{NP}]}=\frac{\frac{\mathrm{Ag} \mathrm{mol}}{L}}{\frac{N P \mathrm{~mol}}{L}}=\frac{\mathrm{Ag} \mathrm{mol}}{N P \mathrm{~mol}}=\frac{\frac{\mathrm{Ag} \mathrm{mol}}{N_{A}}}{\frac{N P \mathrm{~mol}}{N_{A}}}=\frac{\text { Ag atoms }}{N P} \quad \text { Equation } 7
$$

We estimated that synthesized nanoparticles have around $15300 \mathrm{Ag}$ atoms per nanoparticle, and as we have previously demonstrated, our nanoparticles present a $\mathrm{Ag}_{2} \mathrm{~S}$ monoclinic structure, meaning the Ag:S 
ratio in the core is 2:1. Therefore, the core of the nanoparticle will be composed of $15300 \mathrm{Ag}$ atoms and $7650 \mathrm{~S}$ atoms.

In order to confirm our experimental results, the theoretical number of atoms of a pure, structurally perfect $\mathrm{Ag}_{2} \mathrm{~S}$ nanoparticle of the same size was calculated. Firstly, we calculated the theoretical number of unit cells in the NP dividing the total volume of the NP by the volume of the $\alpha$-monoclinic $\operatorname{Ag}_{2} \mathrm{~S}$ unit cell. Then, multiplying the number of unit cells by the number of $\mathrm{Ag}$ and $\mathrm{S}$ atoms inside each cell, the theoretical NP was obtained. Results showed that the theoretical number of atoms is 7.5 times greater than the calculated according our experimental results. It is expected that the experimental number of atoms present in the synthesized NPs was smaller than the number of atoms in the theoretical NP, due to defects in the structure and impurities. Hence, it can be concluded that the estimation of the number of silver atoms per NP is reliable.

As aforementioned, the first population of the fractogram presents an Ag:S molar ratio of 1:1, and corresponds to $\mathrm{Ag}_{2} \mathrm{~S}$ NPs, where the molar ratio for $\mathrm{Ag}: \mathrm{S}$ is 2:1. Hence, it is obvious that there is an excess of sulphur coming from 3-MPA shell and contributing to the 1:1 Ag:S molar ratio. Since each 3MPA molecule has one sulphur atom in its structure, and each NP contains $15300 \mathrm{Ag}$ atoms and $7650 \mathrm{~S}$ atoms, it can be concluded that each NP presents 7650 molecules of 3-MPA in its surface. Moreover, since the diameter of the nanoparticles has been measured with TEM, the surface ligand density can be also estimated, giving rise a value of 152 molecules of 3-MPA $/ \mathrm{nm}^{2}$.

A second significant population was obtained in the AF4 separation of the NPs, which presents a 1:2 $\mathrm{Ag}: \mathrm{S}$ molar ratio and higher size (higher particles present higher elution times). The high ratio of Sulphur is attributed to a bilayer of 3-MPA, where the second layer is composed of two molecules of MPA for each 3-MPA molecule present in the first layer, and the elution time is due to the increase on the final size of the nanoparticle (core and bilayer ligand shell).

\section{Effect of $\mathrm{CN}$ on the luminescence response and proposed mechanism}

It has been widely reported that the interaction of different chemical species with the surface of colloidal QDs produces changes in the emission properties of such nanomaterials, and it is attributed to the surface atoms of the nanoparticle and their close environment [16,17]. In fact, previous research studies carried out in our laboratory demonstrated the different effects that metallic cations [18], inorganic anions $[19,20]$, or organic vapours [21], produced on the emission of luminescent inorganic nanoparticles. In all these applications, the mechanism of interaction mainly depends on the reactive species involved, including electrostatic interaction of the target analyte with the QDs surface ligands [22], electron transfer from conductive band of QD to unoccupied molecular orbital of the analyte [23], or a simple adsorption of the analyte onto the surface of the nanoparticles [24].

However, most of the research applications towards the development of novel optical sensors based on QDs probes are based on changes produced on the luminescence emission in the UV-Vis region. This fact presents disadvantages in certain applications, such as environmental sensing, where the presence of fluorescent compounds, such as pesticides (typically with excitation and emission wavelengths in the UV 
and Vis region, respectively), could interfere with the spectral characteristics of the sensing probe. Moreover, in vitro or in vivo optical sensing, where the tissues absorb part of the excitation light, or where the cell autofluorescence might interfere with the emission of the nanoparticles, is also negatively affected when using nanoparticles with remarkable optical properties in the UV-Vis region. Therefore, the development of NIR emitting QDs is gaining an increasing interest since it has advantages for many different applications.

The synthetized NIR emitting $\mathrm{Ag}_{2} \mathrm{~S}$ QDs emit fluorescence at $795 \mathrm{~nm}$ in aqueous solution that is quenched dose-dependently and selectively in presence of ultratrace levels of cyanide ions. Figure 6 shows the effect of increasing concentrations of free cyanide in aqueous solution on the fluorescence emission of the synthesized $\mathrm{Ag}_{2} \mathrm{~S}$ QDs. Moreover, our experimental results show that rising cyanide concentrations produce a red-shift on the maximum emission wavelength. The observed photoluminescence quenching suffered by the NPs in the presence of cyanide can be explained considering the high dependence of the luminescence emission from the quantum dots to changes in their surface state $[20,25]$. Considering that cyanide has a high affinity to silver association, it is envisaged that cyanide ions would interact strongly with the surface of the Ag-based nanoparticles producing an electron-hole recombination that gives rise to the observed changes in the luminescence. This has been already described for other sensor systems for small analytes based on NP luminescence quenching after direct interaction of the NP with the analyte $[18,26]$.

$<$ Figure 6 near here>

\section{Analytical performance characteristics}

The analytical performance characteristics of the developed methodology were evaluated after optimizing experimental conditions. Calibration graphs were obtained analyzing cyanide standard solutions at increasing concentration by triplicate. In a homogeneous medium, the quencher concentration can be directly related to the luminescence intensity by the Stern-Volmer equation:

$$
\frac{I_{0}}{I}=1+K_{s v}[Q]
$$

where $\mathrm{I}_{0}$ is the intensity of the luminescence of the $\mathrm{Ag}_{2} \mathrm{~S}$ QDs in absence of quencher, $\mathrm{I}$ is the intensity in presence of the quencher, Ksv is the Stern-Volmer constant, and [Q] is the concentration of cyanide. Results obtained proved to be linear up to $1000 \mu \mathrm{M}$ of cyanide (maximum concentration assayed).

The limit of detection (LOD) and the Limit of Quantification (LOQ), calculated as the concentration of cyanide producing an analytical signal equal to three times (LOD) or ten times (LOQ) the standard deviation of 10 injections of a blank, $I=I_{0}-3 \sigma_{\text {blank }}$, turned out to be $1.3 \times 10^{-8} \mathrm{M}, 4.3 \times 10^{-8} \mathrm{M}$ respectively. It is worth to note the LOQ obtained complies with the strict regulations of the European Union, where the maximum tolerable level of cyanide in drinking water is set at $1.9 \times 10^{-6} \mathrm{M}$, but also with the most restrictive tolerable levels set by the Australian and New Zealand Environmental and Conservation Council, set at $7.7 \times 10^{-8} \mathrm{M}$. The precision of the methodology was evaluated in terms of 
repeatability as the relative standard deviation (RSD) of three solutions containing a known concentration of cyanide. For this purpose, three replicates of two different levels of cyanide concentration were evaluated $\left(0.5 \times 10^{-8} \mathrm{M}\right.$ and $\left.5 \times 10^{-7} \mathrm{M}\right)$, giving rise to a low RSD $\leq 2.2$ in all cases $(\mathrm{n}=3$ for each concentration).

The selectivity of the proposed methodology was assessed by studying the effect of the presence of other species that might co-occur in natural water samples on the fluorescence signal. In this sense, anions such as $\mathrm{F}^{-}, \mathrm{Cl}^{-}, \mathrm{Br}^{-}, \mathrm{I}^{-}, \mathrm{NO}_{3}{ }^{-}, \mathrm{NO}_{2}{ }^{-}, \mathrm{SO}_{4}{ }^{2-}, \mathrm{SO}_{3}{ }^{2-}, \mathrm{S}_{2} \mathrm{O}_{3}{ }^{2-}, \mathrm{SCN}^{-}, \mathrm{PO}_{4}{ }^{3-}, \mathrm{S}^{2-}$, and cations including $\mathrm{K}^{+}, \mathrm{Zn}^{2+}, \mathrm{Mg}^{2+}$, $\mathrm{Cu}^{2+}, \mathrm{Na}^{+}, \mathrm{Fe}^{2+}, \mathrm{Ca}^{2+}, \mathrm{Mn}^{2+}, \mathrm{Hg}^{2+}, \mathrm{Cr}^{6+}, \mathrm{As}^{3+}$, were evaluated at increasing concentrations up to $1000 \mu \mathrm{M}$. Results obtained showed that the presence of most of the relevant ions evaluated did not produce any significant effect on the emission of the synthesized $\mathrm{Ag}_{2} \mathrm{~S}$ QDs, even when they are present at $1000 \mu \mathrm{M}$ (the maximum concentration assayed).

However, some ions showed an interferent effect only when present at such very high concentration. Hence, Changes in fluorescence emission were further studied measuring fluorescence of a QD solution containing both, cyanide and the potential interferent at $100 \mu \mathrm{M}$. Results showed that $\mathrm{I}^{-}, \mathrm{Ca}^{2+}$ and $\mathrm{Zn}^{2+}$ caused almost negligible changes in luminescence output below $\pm 3 \%, \mathrm{Mg}^{2+}$ and $\mathrm{S}^{2-}$ produced a slight change in luminescence around $\pm 4 \%$. However, $\mathrm{Cu}^{2+}$ and $\mathrm{Fe}^{3+}$ produced noticeable changes in the emission of the $\mathrm{Ag}_{2} \mathrm{~S}$ QDs over $\pm 30 \%$.

It is worth to note that, except for $\mathrm{Mg}^{2+}$, these ions are typically present at concentrations much lower than those at which they start to interfere (e.g. $\mathrm{Cu}^{2+}: 31.5 \mu \mathrm{M} ; \mathrm{S}^{2-}: 15.6 \mu \mathrm{M}$ ) [11], so it is not expected to find a negative effect attributed to the presence of these ions when analyzing natural drinking water. To avoid these interferences, different strategies based on ions complexion were evaluated. EDTA (0.5 mM) was added to the QD solution in order to overcome this interference, reducing the interference of $\mathrm{Mg}^{2+}$ to levels up to $300 \mu \mathrm{M} \mathrm{Mg}^{2+}$ in the presence of $\mathrm{CN}^{-}$. On the other hand, iron can be complexed with fluoride ion, producing $\mathrm{FeF}_{6}{ }^{3-}$, which does not interfere at concentrations up to $100 \mu \mathrm{M}$. Additionally, taking into account that the limit of detection of the proposed methodology for the determination of cyanide is $1.1 \times 10^{-9} \mathrm{M}$, and that the maximum allowed concentration of cyanide in drinking water in the European Union is $1.9 \times 10^{-6} \mathrm{M}$ (three orders of magnitude higher than the LOD), a potential interferent effect can be easily overcome just by diluting the sample. Then, the concentration of the potential interferent would be lower, but the concentration of cyanide could still be detected with the highly sensitive proposed methodology.

Finally, besides the high sensitivity achieved using $\operatorname{Ag}_{2} \mathrm{~S}$ QDs as sensing probes, it is worth to mention that the NIR emission of these QDs is a great advantage, because the presence of fluorescent compounds, such as pesticides, or any other fluorescent compound whose excitation and emission wavelengths are located in the UV and Vis region, respectively, would not interfere with the spectral characteristics of the developed sensing probe. 
The usefulness of the developed methodology for determination of $\mathrm{CN}^{-}$in natural and bottled water was evaluated following the proposed procedure: $50 \mu \mathrm{L}$ of the $\mathrm{Ag}_{2} \mathrm{~S}$ QDs solution were mixed with $300 \mu \mathrm{L}$ of TRIZMA buffer $\mathrm{pH}=9$ and $300 \mu \mathrm{L}$ of the water sample without any sample pre-treatment, and luminescence measurements were performed. For the application to cyanide sensing, a methodological calibration was always performed for each batch of synthesized nanoparticles, ensuring that eventual small differences in luminescence output from the NPs from batch to batch do not affect the analytical results.

Two different bottled water samples were tested, and since no cyanide was detected in either of them, they were spiked with a known concentration of cyanide to be then studied by standard additions of cyanide. Moreover, natural water from a local creek was also tested using the same methodology, and results obtained are summarized in Table 2. As it can be observed, good recoveries (105-110\%) for the determination of cyanide were obtained in all samples, indicating the performance characteristics and the validation of the proposed methodology for direct analysis of cyanide in water samples.

Table 2. Real sample analysis: Recovery study of spiked cyanide ion in different types of water. Found values correspond to $\mathrm{n}=5$ measurements.

\begin{tabular}{|l|c|c|}
\cline { 2 - 3 } \multicolumn{1}{c|}{} & $\begin{array}{c}{[\mathbf{C N}-] \text { added }} \\
(\mu \mathbf{M})\end{array}$ & $\begin{array}{c}{[\mathbf{C N}-] \text { found }} \\
(\mu \mathbf{M})\end{array}$ \\
\hline Ultrapure (Type I) Water & 0.150 & $0.157 \pm 0.004$ \\
\hline Bottled Water \#1 & 0.020 & $0.021 \pm 0.002$ \\
\hline Bottled Water \#2 & 0.020 & $0.022 \pm 0.001$ \\
\hline Natural Water from a local creek & 0.100 & $0.110 \pm 0.002$ \\
\hline
\end{tabular}

\section{Conclusions}

Water-stabilized 3-MPA $\mathrm{Ag}_{2} \mathrm{~S}$ QDs were synthesized and characterized to determine shape, size, aggregation complexation and concentration. They showed excellent optical properties, emitting light in the NIR region with a very narrow spectrum upon excitation also in the NIR spectral region with a very high QY (33.2\%).

Complementary techniques were required to provide all the information necessary to characterize the synthesized NPs and to optimize their purification process. It was observed that an appropriate purification of the nanoparticles using an ultracentrifugation filtering device was needed to provide narrow emission spectra and a homogenous nanoparticle population, as well as to remove potential agglomerated NPs that would affect negatively to quantitative analytical applications of the QDs. 
Moreover, the combination of complementary techniques such as XRD, TEM and AF4-ICP-MS, have made possible to estimate the nanoparticle number concentration and the surface ligand density, both not easily obtained parameters that are key for many bioanalytical applications. In particular, these parameters are the most important in order to be able to accurately reproduce experiments, because the surface ligand density affects to the interactions of the NPs with their environment, and it is responsible of the colloidal stability of the QDs, and related to unspecific adsorptions, cellular uptake, interactions of the nanoparticle with macrophages, etc. Therefore, the findings here provided are of great value for scientists using nanoparticles in different research application areas.

Finally, the capability of performing analytical sensing of an analyte that is environmentally interesting, based on direct interaction of the analyte with the surface of the QDs has been demonstrated. The synthesized QDs have been evaluated as probes for the detection of cyanide anion in water samples by its quenching effect on the fluorescence of NIR-Emitting QDs. Results have shown that the proposed methodology is rapid, selective, extremely sensitive and works on the basic $\mathrm{pH}$ range which is of great value for the detection of $\mathrm{CN}^{-}$, giving rise to very good analytical performance characteristics for the detection of cyanide in natural waters.

It must be highlighted that the here synthesized 3-MPA $\operatorname{Ag}_{2} \mathrm{~S}$ QDs have shown excellent optoelectronic properties, with maximum excitation and emission wavelengths in the NIR region, are stable in aqueous media and do not contain toxic heavy metals. Such advantageous optical behaviour is of enormous importance in several fields, including bioimaging, since the autofluorescence of cells or tissues that takes place in the UV-Vis region is avoided, and a more efficient penetration of the excitation source is achieved; or in environmental applications, where the presence of other compounds that emit fluorescence in the UV-Vis region does not affect the signal of the NIR $\mathrm{Ag}_{2} \mathrm{~S}$ QDs. Further research using these nanoparticles would take advantage of the $\mathrm{Ag}_{2} \mathrm{~S}$ QDs NIR capabilities for their use in complex biological matrices and bioimaging. Particularly the use of $\operatorname{Ag}_{2} \mathrm{~S}$ NIR QDs is being explored in our laboratory as antibody luminescent labels for the development of immunoassays specific of selected antigens in biological samples.

\section{Acknowledgements}

Financial support provided from Project CTQ2016-79412-P (Ministerio de Economia y Competitividad, Spain) and RTA2015-0061 (Instituto Nacional de Investigaciones Agrarias) is gratefully acknowledged. Diego Bouzas-Ramos acknowledges the PhD grant BP14-137 from Principado de Asturias, Spain.

\section{Figure captions}

Figure 1 HRTEM image of the synthesized $\mathrm{Ag}_{2} \mathrm{~S}$ QDs indicating the lattice distances measured in nanoparticles at different planes, which are in agreement with theoretical $\alpha$-monoclinic $\operatorname{Ag}_{2} \mathrm{~S}$ structure $(\mathrm{a}=4.23 \AA, \mathrm{b}=6.91 \AA$, and $\mathrm{c}=8.29 \AA)$. 
Figure 2 FTIR spectrum of the NIR $\mathrm{Ag}_{2} \mathrm{~S}$ QDs.

Figure 3 XRD spectra shows four scans of the sample performed at different times, where no differences are noticeable, thus indicating the stability of the sample after a 24 hour drying step at $180^{\circ} \mathrm{C}$.

Figure 4 Absorption and luminescence spectra of the synthesized $\mathrm{Ag}_{2} \mathrm{~S}$ Quantum Dots. The absorption band (blue line) shows a shoulder with a peak centered at $750 \mathrm{~nm}$. Luminescence excitation with $\lambda_{\mathrm{ex}}$ maximum at $750 \mathrm{~nm}$ (grey line), and emission band (orange line) in the NIR region $\left(\lambda_{\mathrm{em}} 800 \mathrm{~nm}\right)$.

Figure 5 Monitorization of the ${ }^{107} \mathrm{Ag}$ signal using AF4 coupled on line to ICP-MS. Grey fractogram represents the unpurified QDs, while the black fractogram represents the QDs obtained after purification with an Amicon filter

Figure 6 Effect of increasing concentrations of cyanide on the luminescence of $\operatorname{Ag}_{2} \mathrm{~S}$ QDs: (up to down: $0,120,800$ and $1000 \mu \mathrm{M}$ of cyanide).

\section{FIGURES}
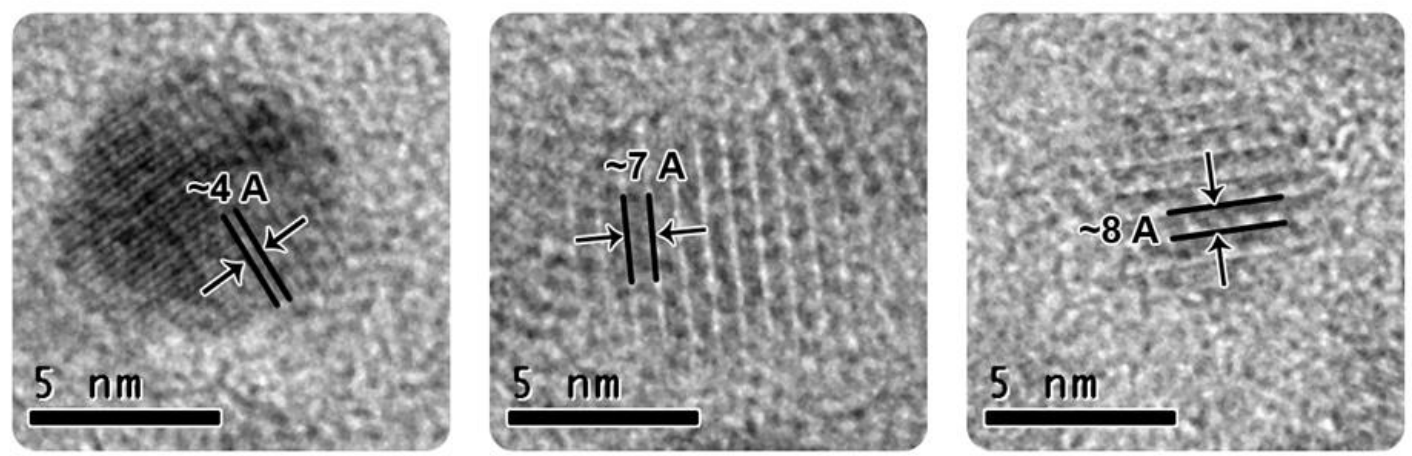

Figure 1 


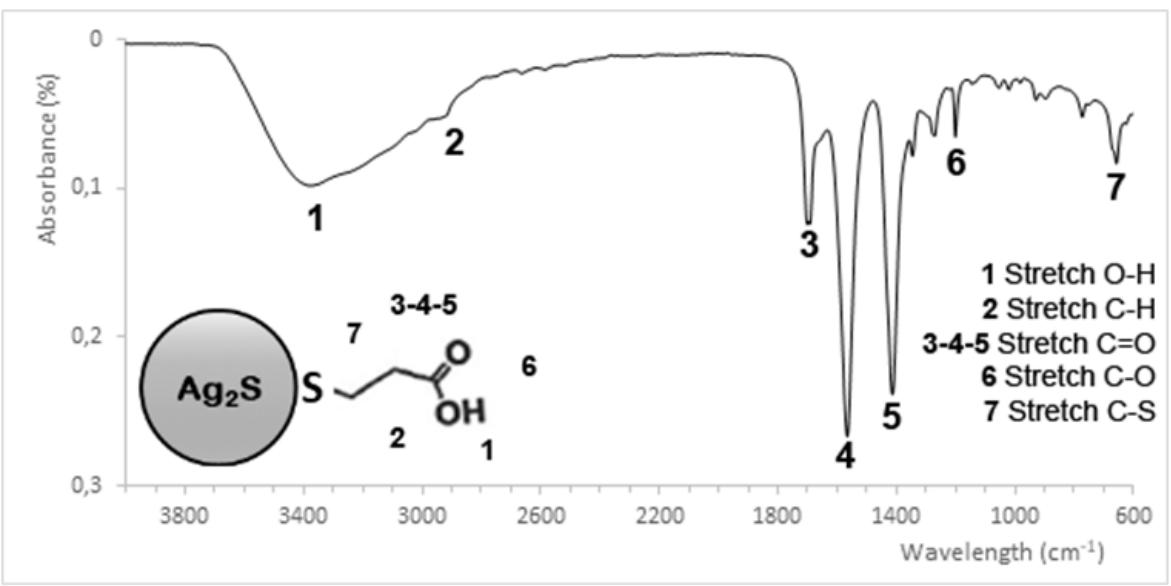

Figure 2

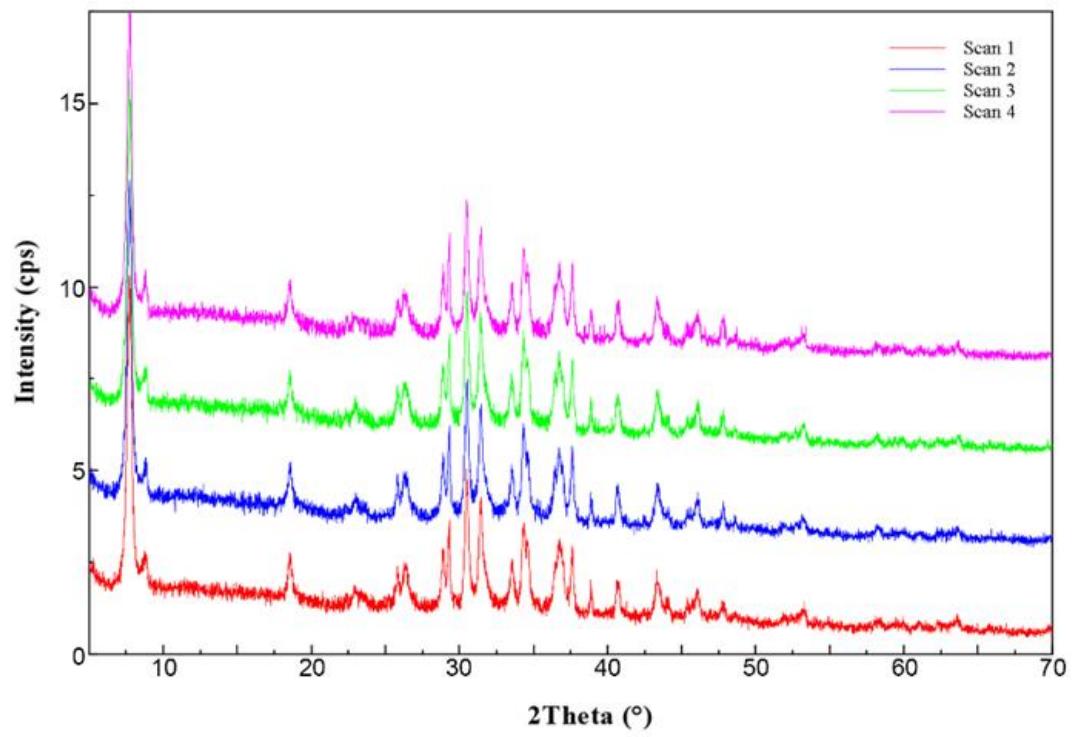

Figure 3 


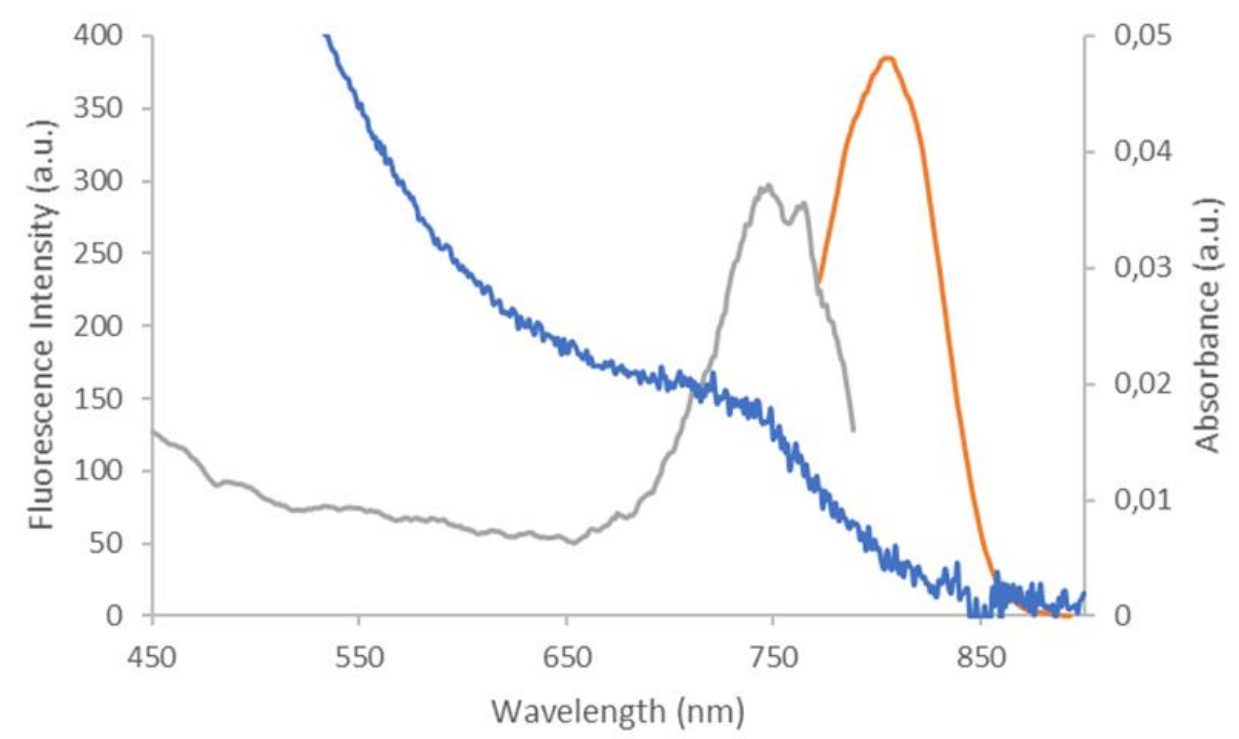

Figure 4

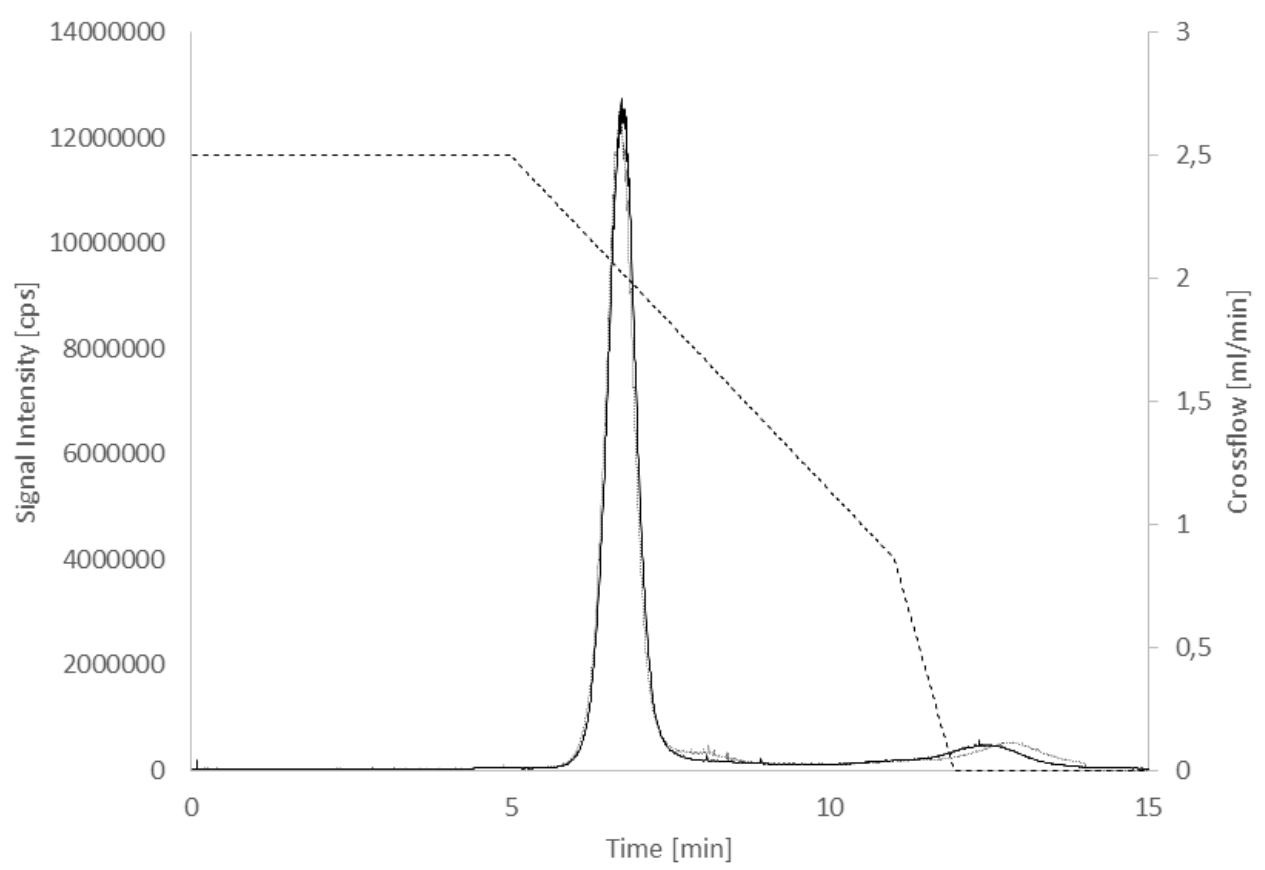

Figure 5 


\section{References}

[1] A.P. Alivisatos, Semiconductor Clusters, Nanocrystals, and Quantum Dots, Science 271 (1996) 933937. https://doi.org/10.1126/science.271.5251.933

[2] C.B. Murray, C.J. Norris, M.G. Bawendi, Synthesis and characterization of nearly monodisperse CdE (E = sulfur, selenium, tellurium) semiconductor nanocrystallites, J. Am. Chem. Soc. 115 (1993) 87068715. https://doi.org/10.1021/ja00072a025

[3] S. Xu, J. Cui, L. Wang, Recent developments of low-toxicity NIR II quantum dots for sensing and bioimaging, Trends Anal. Chem. 80 (2016) 149-155. https://doi.org/10.1016/j.trac.2015.07.017

[4] Y.P. Gu, R. Cui, X.L. Zhang, Z.X. Xie, D.W. Pang, Ultrasmall near-infrared Ag2Se quantum dots with tunable fluorescence for in vivo imaging, J. Am. Chem. Soc., 134 (2012) 79-82. https://doi.org/10.1021/ja2089553

[5] M. Menéndez, D. Bouzas, J. Ruiz, J.M. Costa-Fernandez, A. Sanz-Medel, Mass spectrometry for the characterization and quantification of engineered inorganic nanoparticles. Trends Anal. Chem. 84 (2016) 139-148. https://doi.org/10.1016/j.trac.2016.06.001

[6] M. García-Cortes, E. Sotelo, M.T. Fernandez-Arguelles, J. Ruiz, J.M. Costa-Fernandez, A. SanzMedel, Capping of Mn-Doped ZnS quantum dots with DHLA for their stabilization in aqueous media: determination of the nanoparticle number concentration and surface ligand density. Langmuir 33 (2017) 6333-6341. https://doi.org/10.1021/acs.langmuir.7b00409

[7] World Health Organization. 'Concise international chemical assessment document 61, Hydrogen cyanide and cyanides: human health aspects'. Geneva: 2004 [Accessed December 12, 2017]. p. 4-5. http://www.who.int/ipcs/publications/cicad/en/cicad61.pdf

[8] World Health Organization. ‘Guidelines for Drinking-Water Quality. Fourth Edition'. Geneva, 2011 [Accessed December 12, 2017]. p. 343.

http://apps.who.int/iris/bitstream/10665/44584/1/9789241548151_eng.pdf

[9] United States Enviromental Protection Agency. 'Human Health - water ingestion only Fact Sheet for Cyanide: (Human Health Noncarcinogen - water ingestion only)', New York State Department of Environmental Conservation (PDF): 1998 [Accessed December 12, 2017] p. 1. https://www.epa.gov/sites/production/files/2015-06/documents/ny_hh_359_w_03121998.pdf

[10] Health Canada. 'Guidelines for Canadian Drinking Water Quality: Guideline Technical Document Cyanide’. Canada: 1991 [Accessed December 12, 2017] p. 1. https://www.canada.ca/en/healthcanada/services/publications/healthy-living/guidelines-canadian-drinking-water-quality-guidelinetechnical-document-cyanide.html

[11] European Drinking Water Directive. 'Council Directive 98/83/EC of 3 November 1998 on the quality of water intended for human consumption'. 1998 [Accessed December 12, 2017]. p. 11. http://eurlex.europa.eu/legal-content/EN/TXT/PDF/?uri=CELEX:31998L0083\&from=EN

[12] Australian and New Zealand Environmental and Conservation Council (ANZECC). Agriculture and Resource Management Council of Australia and New Zealand. 'Australian Water Quality Guidelines for Fresh and Marine Water'; 2000 [Accessed December 12, 2017]. p. 3-5.

http://www.mincos.gov.au/_data/assets/pdf_file/0019/316126/wqg-ch3.pdf 
[13] I. Hocaoglu, M.N. Çizmeciyan, R. Erdem, C. Ozen, A. Kurt, A. Sennaroglu, H.Y. Acar, Development of highly luminescent and cytocompatible near-IR-emitting aqueous $\mathrm{Ag}_{2} \mathrm{~S}$ quantum dots. $\mathrm{J}$. Mater. Chem. 22 (2012) 14674-14681. https://doi.org/10.1039/c2jm31959d

[14] J. Coates, Interpretation of Infrared Spectra, A Practical Approach, Encyclopedia of Analytical Chemistry, R.A. Meyer (Ed.) Copyright, 2000 John Wiley \& Sons, Ltd. https://doi.org/10.1002/9780470027318.a5606

[15] A.T.R. Williams, S.A. Winfield, J.N. Miller, Relative fluorescence quantum yields using a computercontrolled luminescence spectrometer. Analyst 108 (1983) 1067-1071.

https://doi.org/10.1039/AN9830801067

[16] J.M. Costa-Fernandez, R. Pereiro, A. Sanz-Medel, The use of luminescent quantum dots for optical sensing. Trends Anal.Chem. 25 (2006) 207-218. https://doi.org/10.1016/j.trac.2005.07.008

[17] L. Qu, X. Peng, Control of Photoluminescence Properties of CdSe Nanocrystals in Growth. J. Am. Chem. Soc. 124 (2002) 2049-2055. https://doi.org/10.1021/ja017002j

[18] M.T. Fernandez-Argüelles, W.J. Jin, J.M. Costa-Fernandez, R. Pereiro, A. Sanz-Medel, Surfacemodified CdSe quantum dots for the sensitive and selective determination of $\mathrm{Cu}(\mathrm{II})$ in aqueous solutions by luminescent measurements. Anal. Chim. Acta 549 (2005) 20-25.

https://doi.org/10.1016/j.aca.2005.06.013

[19] W.J. Jin, M.T. Fernandez-Argüelles, J.M. Costa-Fernandez, R. Pereiro, A. Sanz-Medel, Photoactivated luminescent CdSe quantum dots as sensitive cyanide probes in aqueous solutions. Chem. Commun. 7 (2005) 883-885. https://doi.org/10.1039/b414858d

[20] N. Vasimalai, M.T. Fernandez-Argüelles, Novel one-pot and facile room temperature synthesis of gold nanodots and application as highly sensitive and selective probes for cyanide detection, Nanotechnology, 27 (2016) 475505. https://doi.org/10.1088/0957-4484/27/47/475505

[21] E. Sotelo-Gonzalez, A.M. Coto-Garcia, M.T. Fernandez-Argüelles, J.M. Costa-Fernandez, A. SanzMedel, Immobilization of phosphorescent quantum dots in a sol-gel matrix for acetone sensing, Sensors and Actuators B: Chemical, 174 (2012) 102-108. DOI: 10.1016/j.snb.2012.07.107

[22] J. Chen, A. Zheng, Y. Gao, C. He, G. Wu, Y. Chen, X. Kai, C. Zhu, Functionalized CdS quantum dots-based luminescence probe for detection of heavy and transition metal ions in aqueous solution. Spectrochim. Acta Part A 69 (2008) 1044-1052. https://doi.org/10.1016/j.saa.2007.06.021

[23] R. Tu, B. Liu, Z. Wang, D. Gao, F. Wang, Q. Fang, Z. Zhang, Amine-Capped ZnS-Mn2+ Nanocrystals for Fluorescence Detection of Trace TNT Explosive. Anal. Chem. 80 (2008) 3458-3465. https://doi.org/10.1021/ac800060f

[24] A.V. Isarov, J. Chrysochoos, Optical and Photochemical Properties of Nonstoichiometric Cadmium Sulfide Nanoparticles: Surface Modification with Copper(II) Ions. Langmuir 13 (1997) 3142-3149. https://doi.org/10.1021/la960985r

[25] W. J. Jin, J. M. Costa-Fernández, R. Pereiro, A. Sanz-Medel, Surface-modified CdSe quantum dots as luminescent probes for cyanide determination. Analytica Chimica Acta 522 (2004) 1-8. https://doi.org/ 10.1016/j.aca.2004.06.057

[26] C.F. Landes, M. Braun, M. A. El-Sayed, On the Nanoparticle to Molecular Size Transition: Fluorescence Quenching Studies. J. Phys. Chem. B 105 (2001), 10554-10558. https://doi.org/10.1021/jp0118726 